\title{
Facial Expression Recognition in Video with Multiple Feature Fusion
}

\author{
T.Sai Teja, M.K.Mariam Bee
}

\begin{abstract}
Video based outward appearance affirmation has been a long standing issue and pulled in creating thought starting late. The way to a productive outward appearance affirmation system is to manhandle the conceivable outcomes of shifting media modalities and structure incredible highlights to effectively depict the facial appearance and course of action changes achieved by facial developments. We propose a powerful system to address this issue in this paper. In our examination, both visual modalities (face pictures) and sound modalities (talk) are used. Another part descriptor known as bar chart of familiarized Gradients from 3 Orthogonal Planes (HOG-TOP) is projected to free dynamic surfaces from video courses of action to depict facial look changes. In addition, another effective geometric element got from the curve change of facial places of interest is proposed to get facial plan changes.
\end{abstract}

Keywords: Media modalities, visual modalities, sound modalities, HOG-TOP.

\section{INTRODUCTION}

Activity Units (AUs) are the fundamental muscle developments and enhanced visualizations of face activities, which fill in as an integral asset in breaking down complex outward appearances. Facial Action Unit discovery has been contemplated for a considerable length of time and assumes a principal job in psychopathology, social flag preparing and conduct science. Past investigates on $\mathrm{AU}$ location center around geometric highlights and human designed descriptors, for example, HOG, which can't completely uncover trademark portrayal of AUs. As of late, some profound learning calculations have been proposed to adapt better portrayals of AUs. They endeavor to take care of the issue from three perspectives dependent on three qualities for AU discovery: locale meagerly, multi mark learning and transient reliance. Right off the bat, with the earlier information that AUs are dynamic just on meager locales, it is critical to distinguish explicit districts for every AU. Furthermore, there has been solid measurable proof that there are high relationships among AUs. Hence, it bodes well to mutually become familiar with numerous AUs. Thirdly, in a transient succession, current edge's AUs have solid reliance on past and ensuing edges', which implies it's important to join fleeting reliance in AU identification. Be that as it may, late methodologies just think about a couple of viewpoints in $\mathrm{AU}$ recognition and some depend on extra data like milestones. So as to accomplish the best AU location execution, each of the three angles should be considered. Outward appearance, as a ground-breaking nonverbal channel assumes an essential job for people to pass on feelings and transmit messages. Programmed outward appearance acknowledgment (AFEC) can be broadly connected in numerous fields, for example, therapeutic appraisal, lie recognition and human PC association. AFEC has pulled in incredible enthusiasm for as long as two decades. In any case, outward appearance examination is an extremely difficult undertaking since outward appearances brought about by facial muscle developments are inconspicuous and transient. To catch and speak to these developments is a key issue to be tended to in outward appearance investigation. Two standards of outward appearances examination are broadly received in the ebb and flow innovative work. One stream is to identify facial activities. The examination announced in, demonstrated that every outward appearance contains an exceptional gathering of facial activity units. The Facial Action Coding System (FACS), which was first proposed by Ekman and Friesen in 1978 and after that improved in 2002, is the best realized framework produced for people to depict facial activities. Another surge of outward appearance investigation is to do facial effect (feeling) acknowledgment specifically. Most analysts manage the acknowledgment errand of six general feelings: cheerful, pitiful, dread, nauseate, irate and shock. Numerous endeavors have been made for outward appearance acknowledgment. The systems utilized are usually ordered Junkai Chen and Zheru Chi are with the Department of Electronic and Information Engineering, A geometry based strategy catches facial arrangements in which a lot of facial fiducial focuses is utilized to describe the face shape.

\section{THE IMAGE PROCESSING SYSTEM}

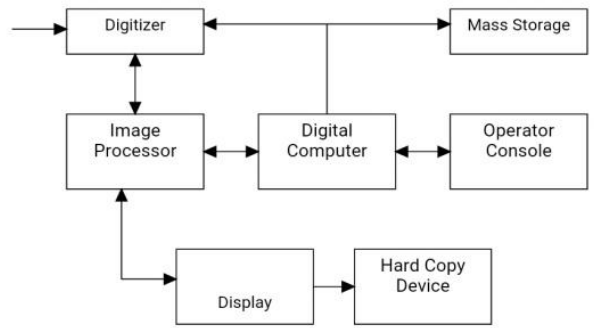

Fig. 1 block diagram of image processing system

\section{DIGITIZER:}

A data converter converts a picture into a numerical illustration appropriate for input into a computer. Some common digitizers are

1. Microdensitometer

2. Flying spot scanner

3. Image dissector

4. Videocon camera

5. Photosensitive solid- state arrays.

Revised Manuscript Received on August 14, 2019.

T.Sai Teja, Department of Electronics \& Communication Saveetha School Of Engineering(SIMATS), Chennai. Tamil Nadu, India. (Email: Saiteja66677@gmail.com)

M.K.Mariam Bee, Department of Electronics \& Communication Saveetha School Of Engineering(SIMATS), Chennai. Tamil Nadu, India. (Email: Mariamresearch09@gmail.com)

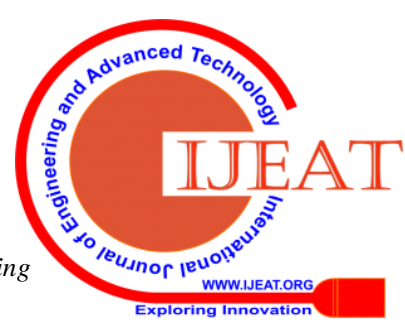




\section{IMAGE PROCESSOR:}

A picture processor does the elements of picture securing, capacity, preprocessing, division, portrayal, acknowledgment and elucidation lastly shows or records the subsequent picture. The accompanying square outline gives the principal succession engaged with a picture preparing framework.

As definite in the outline, the initial phase in the process is picture procurement by an imaging sensor related to a digitizer to digitize the picture. The subsequent stage is the preprocessing step where the picture is improved being encouraged as a contribution to alternate procedures. Preprocessing regularly manages improving, expelling clamor, separating areas, and so forth. Division segments a picture into its constituent parts or items. The yield of division is normally crude pixel information, which comprises of either the limit of the district or the pixels in the locale themselves. Portrayal is the way toward changing the crude pixel information into a structure helpful for ensuing handling by the PC. Portrayal manages extricating highlights that are fundamental in separating one class of items from another. data given by its descriptors. Elucidation includes allotting importance to an outfit of perceived articles. The information about an issue area is consolidated into the learning base. The learning controls the cooperation between the modules. Not all modules need be essentially present for a particular capacity. The synthesis of the picture preparing framework relies upon its application. The edge rate of the picture processor is regularly around 25 outlines for every second.

\section{DIGITAL COMPUTER:}

Scientific preparing of the digitized picture, for example, convolution, averaging, expansion, subtraction, and so forth are finished by the PC.

\section{MASS STORAGE:}

The auxiliary stockpiling gadgets regularly utilized are floppy circles, CD ROMs and so forth.

\section{HARD COPY DEVICE:}

The printed version gadget is utilized to deliver a perpetual duplicate of the picture and for the capacity of the product included.

\section{OPERATOR CONSOLE:}

The administrator reassure comprises of hardware and plans for confirmation of halfway outcomes and for changes in the product as and when require. The administrator is likewise fit for checking for any subsequent blunders and for the passage of essential information.

\section{OBJECTIVE}

Outward appearance, as an amazing nonverbal channel, assumes an imperative job for people to pass on feelings and transmit messages. Programmed outward appearance acknowledgment (AFEC) can be broadly connected in numerous fields, for example, restorative appraisal, lie recognition and human PC collaboration..

\section{EXISTING SYSTEM}

Static picture based techniques or dynamic surface put together strategies just depend with respect to visual modalities. Notwithstanding, sound or discourse is additionally essential for people to pass on feelings and goals. Sound modalities can give some integral data notwithstanding visual modalities. As of late, varying media based strategies for influence acknowledgment have pulled in developing consideration from the emotional figuring Acknowledgment appoints a name to an article dependent on the base aides the activity of each preparing module and furthermore

network. Various methodologies have been proposed to consolidate sound and visual modalities for influence acknowledgment. A far reaching study can be found in. Acoustic highlights removed from voice or discourse and visual highlights separated from face pictures are joined to handle this issue. For instance, voice and lip movement were utilized in. Face pictures and discourse were utilized. The techniques revealed in connected a few element descriptors, for example, SIFT, HOG, PHOG and so on to compose face pictures and consolidated them with acoustic alternatives to recognize facial highlights inside nature.

\section{EXISITING SYSTEM DISADVANTAGES}

1. Past works predominantly centered on static and single face picture based outward appearance acknowledgment. Recently, facial articulation acknowledgment in video has pulled in extraordinary intrigue.

2. Compared with a static picture, a video succession can give spatial appearance as well as include facial movements and went with discourse.

3. The key to tackle the issue of video based outward appearance acknowledgment is to abuse the portrayal capacity of multi modalities (for example visual and sound data) and structure powerful highlights to adequately describe the facial appearance and setup changes brought about by facial solid exercises.

\section{PROPOSED SYSTEM}

1. We build up a system which can viably handle outward appearance acknowledgment in video. A numerous component combination strategy is utilized to manage outward appearance acknowledgment under lab-controlled condition and in the wild, individually.

2. We propose another element descriptor HOG-TOP to characterize facial appearance changes and another viable geometric element to catch facial design changes.

3. We demonstrate that numerous highlights can make diverse commitments and can accomplish preferable execution over the individual highlights connected alone. We additionally demonstrate that numerous component combinations can upgrade the discriminative intensity of different highlights.

\section{BLOCK DIAGRAM}

\section{Imput video}

\section{$+1$}

Fram e comwersinm.

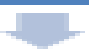

Feature Extraction.

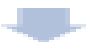

feature Pooling.

\section{$+1$}

Classification.

Fig. 2 block diagram of proposed system 


\section{MODULE DESCRIPTION}

\section{MODULE 1}

\section{Frame Conversion}

Video input converts to outline arrangement.

\section{MODULE 2}

\section{Feature Extraction}

\section{A. Histograms of situated angles}

Histograms of situated angles (HOG) were first proposed for human location. The essential arrangement of HOG is that local article look and structure will commonly be described great by the dispersion of local force inclinations or edge headings. Hoard is touchy to question disfigurements. Outward appearances are brought about by facial muscle developments. For instance, mouth opening and cocked eyebrows will create an unexpected outward appearance. These developments could be viewed as sorts of distortions. Hoard will viably catch and speak to these mishapenings. Be that as it may, the first HOG is constrained to manage a static picture. So as to demonstrate dynamic surfaces from a video grouping with HOG, we stretch out HOG to 3-D to process the arranged angles on three symmetrical planes XY, XT, and YT (TOP), for example Hoard TOP. The arranged HOG-TOP is utilized to describe facial look changes.

\section{B. Geometric element}

In this segment, we will in general present an a great deal of solid geometric element explicitly geometric twist include, which is gotten from the twist change of the facial tourist spots. Outward appearances are brought about by facial muscle developments. These developments lead to the removals of the facial tourist spots. Here we will in general accept that each face picture comprises of the many sub-locales. These sub-areas can be shaped with triangles with their vertexes situated at facial milestones, as appeared in Fig. 5. The removals of facial milestones cause the distortions of the triangles. We propose to use the distortions to speak to facial arrangement changes.

\section{Acoustic Feature}

Visual modalities (face pictures) and sound modalities (discourse) can both pass on the feelings and goals of people. Sound modalities conjointly offer some supportive pieces of information for affect acknowledgment in video. For example, with voice flag, the technique proposed an upgraded autocorrelation (EAC) include for feeling acknowledgment in video.

\section{MODULE 3}

\section{Feature pooling}

Highlights from various modalities can make distinctive commitments. Customary SVM links absolutely \{different completely different $\}$ alternatives into one component vector and structured one part for of these diverse choices. Be that as it may, building a portion for each assortment of choices and integration these pieces ideally will upgrade the discriminative intensity of those alternatives.

\section{MODULE 4}

\section{Classification}

To Construct Associate in Nursing best hyper plane, SVM Employs An Iterative Training Algorithm, Which Is Used To Minimize An Error Function. As indicated by the state Of The Error perform, SVM Models might be Classified Into Four Distinct Groups: Classification SVM kind one (Also called C-SVM Classification).

\section{PROPOSED SYSTEM TECHNIQUE EXPLANATION}

We can see that highlight extraction assumes an inside job on influence acknowledgment in video. Structuring a successful element is vital and significant. LBP-TOP is broadly utilized for displaying dynamic surfaces. Nonetheless, there are two restrictions of LBP-TOP. One is the high dimensionality. The measure of LBP-TOP coded utilizing a uniform example is 59_3. Also, in spite of the fact that LBP-TOP is vigorous to manage brightening transforms, it is obtuse to facial muscle disfigurements. In this work, we propose another element called HOG-TOP, which is increasingly minimized and successful to describe facial appearance changes. Moreover, design and shape portrayals assume an essential job in human vision for the view of outward appearances. We trust that past works have not yet completely misused the possibilities of arrangement portrayals. Portraying face shape or estimating relocations of fiducial focuses, just are not adequate to catch facial arrangement changes, particularly the inconspicuous non-unbending changes. In this work, we present a progressively hearty geometric component to catch facial design changes.

\section{ADVANTAGES}

Experiments directed on the dataset exhibit that our methodology can accomplish a promising act in outward appearance acknowledgment in video.

\section{APPLICATIONS}

Programmed outward appearance acknowledgment (AFEC) can be generally connected in numerous fields, for example, restorative appraisal, lie identification and human PC cooperation.

\section{RESULT AND OUTPUT}

In this section, the performance of the system is evaluated. The input given and output is shown in the fig 3 , fig 4 ,fig5

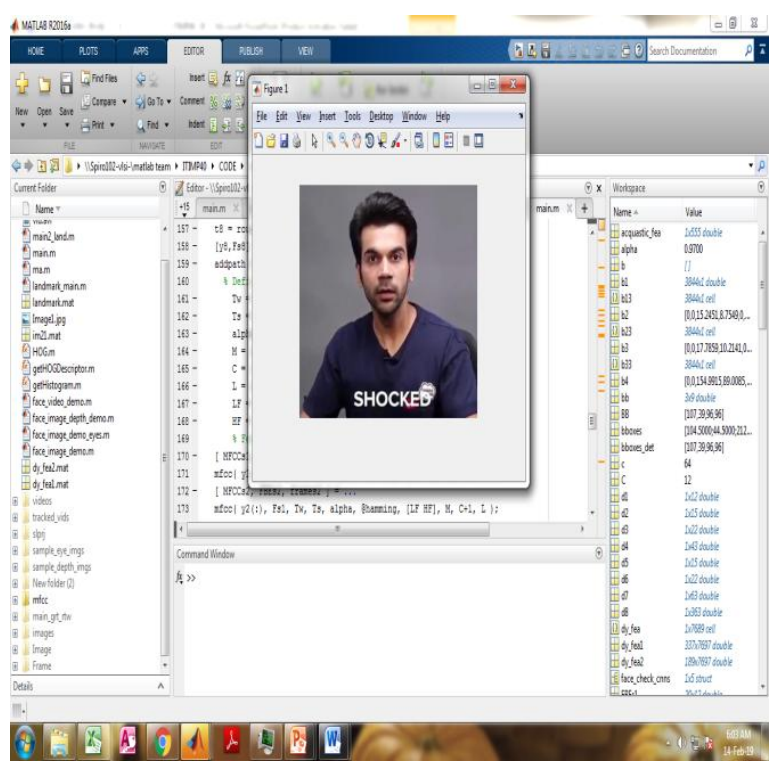

Fig. 3 input images

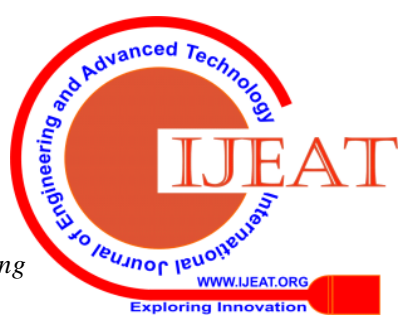




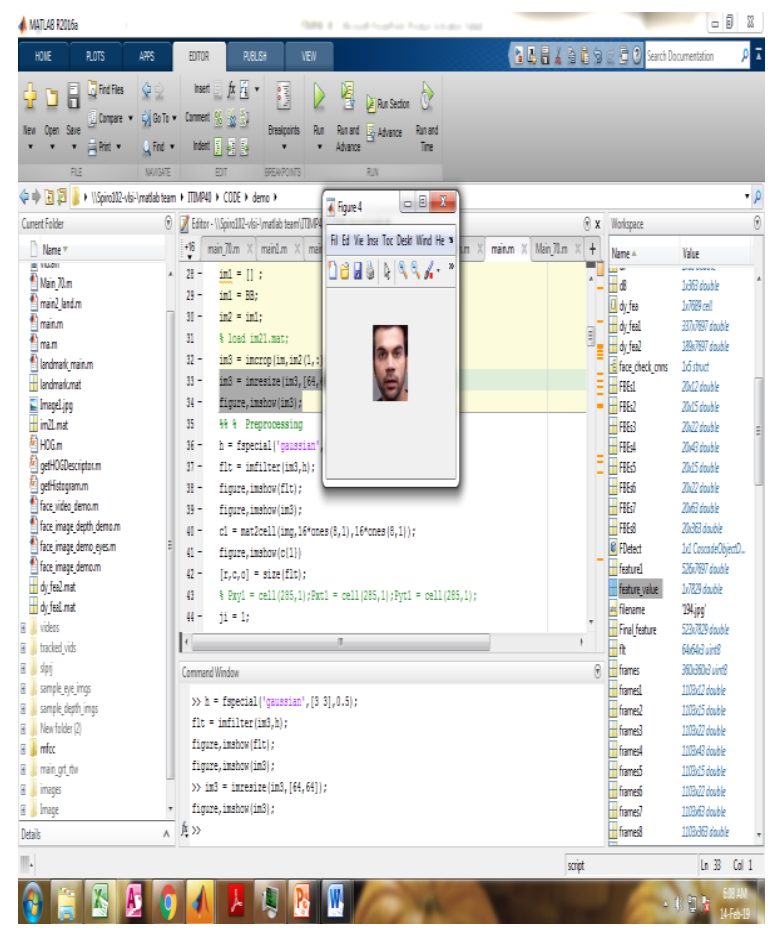

Fig. 4 resized image

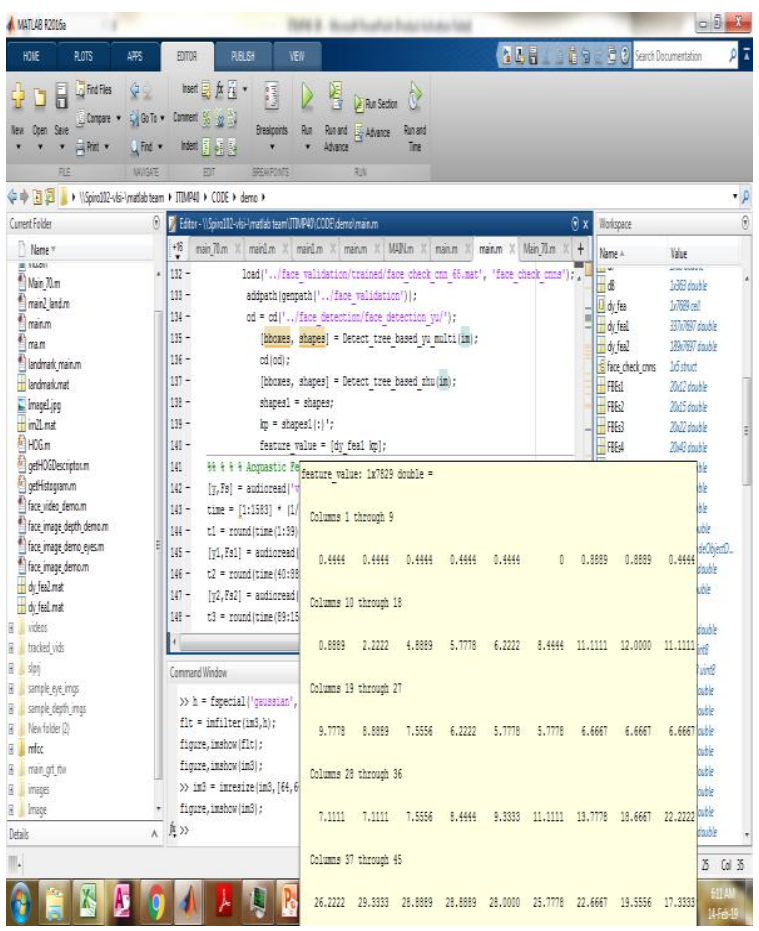

Fig. 5 feature values

\section{CONCLUSION}

Video based outward appearance acknowledgment is a testing and long standing issue. In this paper, we abuse the possibilities of varying media modalities and propose a viable system with different element combination to deal with this issue. Both the visual modalities (face pictures) and sound modalities (discourse) are used in our examination. Another component descriptor called Histogram of Oriented Gradients from Three Orthogonal Planes (HOG-TOP) is proposed to remove dynamic surfaces from video groupings to describe facial appearance changes. Tests directed on three open databases (CK+, GEMEP-FERA 2011, AFEW4.0) have shown that HOG-TOP executes just as a broadly used feature LBPTOP in speaking to dynamic surfaces from video groupings. In addition, HOG-TOP is increasingly viable to catch unobtrusive facial appearance changes and vigorous in managing outward appearance acknowledgment in nature. What's more, HOG-TOP is progressively smaller. So as to catch facial arrange transforms, we present a powerful geometric element getting from the twist change of the facial tourist spots. Understanding that voice is another incredible route for individuals to transmit message, we likewise investigate the job of discourse and utilize the acoustic component for influence acknowledgment in video. We connected the different element combination to manage outward appearance acknowledgment under lab controlled environment and in nature. Analyses led on two outward appearance datasets, CK+ and AFEW 4.0, exhibit that our methodology can accomplish a promising performance in outward appearance acknowledgment in video.

\section{REFERENCES}

1. R. A. Calvo and S. D'Mello," Affect Detection an Interdisciplinary Review of Models, Methods, and Their Applications,"

2. IEEE Transactions on Affective Computing, vol. 1, pp 18-37, 2010.

3. Y. 1. Tian, T. Kanade, and J. F. Cohn, "Recognizing action units for facial expression analysis," IEEE Transactions on Pattern Analysis and Machine Intelligence, vol. 23, pp. 97-115, 2001.

4. K. Scherer and P. Ekman,"Handbook of Methods in Nonverbal Behavior Research," UK: Cambridge Univ. Press, 1982.

5. J. F. Cohn and P. Ekman,"Measuring facial action," 2005.

6. P. Ekman and W. V. Friesen,'Facial Action Coding System: A Technique for the Measurement of Facial Movement," Consulting Psychologists Press, 1978.

7. P. Ekman, W. V. Friesen, and J. C. Hager, "Facial Action Coding System: The Manual on CD ROM. A Human Face," 2002.

8. P. Ekman,"An argument for basic emotions," Cognition \& Emotion, vol. 6, pp. 169-200, 1992.

9. S. Z. Li and A. K. Jain, "Handbook of face recognition," springer, 2011.

10. N. Dalal and B. Triggs, "Histograms of Oriented Gradients for Human Detection," IEEE Conference on Computer Vision and Pattern Recognition, 2005, pp 886-893.

11. G. Zhao and M. Pietikainen,'Dynamic texture recognition using local binary patterns with an application to facial expressions," IEEE Transactions on Pattern Analysis and Machine Intelligence, vol. 29, pp. 915-928, 2007. 